\title{
FORMAL BIHOLOMORPHIC MAPS OF REAL ANALYTIC HYPERSURFACES
}

\author{
NORDine Mir
}

\begin{abstract}
Let $f:(M, p) \rightarrow\left(M^{\prime}, p^{\prime}\right)$ be a formal biholomorphic mapping between two germs of real analytic hypersurfaces in $\mathbb{C}^{n}, p^{\prime}=f(p)$. Assuming the source manifold to be minimal at $p$, we prove the convergence of the so-called reflection function associated to $f$. As a consequence, we derive the convergence of formal biholomorphisms between real analytic minimal holomorphically nondegenerate hypersurfaces. Related results on partial convergence of formal biholomorphisms are also obtained.
\end{abstract}

\section{Introduction}

A formal (holomorphic) mapping $f:\left(\mathbb{C}^{n}, p\right) \rightarrow\left(\mathbb{C}^{n}, p^{\prime}\right), p, p^{\prime} \in \mathbb{C}^{n}, n \geq 1$, is a vector $\left(f_{1}(z), \ldots, f_{n}(z)\right)$ where each $f_{j}(z) \in \mathbb{C}[[z-p]]$, the ring of formal holomorphic power series in $z-p$, and $f(p)=p^{\prime}$. The mapping $f$ is called a formal biholomorphism if its Jacobian does not vanish at $p$. If $M, M^{\prime}$ are two smooth real real-analytic hypersurfaces in $\mathbb{C}^{n}$ through $p$ and $p^{\prime}$ respectively, we say that a formal mapping $f$ as above sends $M$ into $M^{\prime}$ if $\rho^{\prime}(f(z), \overline{f(z)})=$ $a(z, \bar{z}) \rho(z, \bar{z})$, where $\rho, \rho^{\prime}$ are local real-analytic defining functions for $(M, p)$ and $\left(M^{\prime}, p^{\prime}\right)$ respectively and $a \in \mathbb{C}[[z-p, \bar{z}-\bar{p}]]$. In this paper we study the convergence (and partial convergence) of formal biholomorphic mappings between germs of real analytic hypersurfaces in $\mathbb{C}^{n}$ in terms of optimal and natural geometric conditions on the source and target manifolds.

A natural geometric condition which appears in this regularity problem is the concept of holomorphic nondegeneracy. Following Stanton, a real analytic hypersurface $M \subset \mathbb{C}^{n}$ is called holomorphically nondegenerate if, near any point in $M$, there is no non-trivial holomorphic vector field, with holomorphic coefficients, tangent to $M$ near that point [16, 2]. Baouendi and Rothschild [7] recognized the importance of such a condition and used it to characterize those real algebraic hypersurfaces for which any biholomorphic self-map must be algebraic (see also [6]). In this paper we establish a similar statement for formal biholomorphic mappings of real analytic hypersurfaces (Theorem 2.2 below), namely that any formal biholomorphism between germs of real analytic minimal and holomorphically nondegenerate hypersurfaces is convergent. This statement will

Received February 11, 2000.

2000 Mathematics Subject Classification. 32H99, 32H40, 32 V35.

Key words and phrases. Formal mapping, Real analytic hypersurfaces, Holomorphic nondegeneracy, Cauchy estimates, Artin approximation theorem. 
in fact be a consequence of our main result, Theorem 2.1, where a description of the analyticity properties of formal biholomorphic maps of minimal real analytic hypersurfaces is given. An application of this theorem to partial convergence of such maps is also given in $\S 7$.

The study of the convergence of formal mappings between real analytic CR submanifolds goes back to Chern and Moser [9], who established the convergence of formal biholomorphisms between real analytic Levi-nondegenerate hypersurfaces. More recently, Baouendi, Ebenfelt and Rothschild [3, 4, 5] addressed this problem in more general situations. In particular, the following two facts follow from their work:

i) Given a generic minimal real analytic connected holomorphically nondegenerate submanifold $M$ in $\mathbb{C}^{N}, N \geq 2$, there exists a proper real analytic subvariety $S \subset M$, such that for any point $p \in M \backslash S$, any formal biholomorphism sending $(M, p)$ onto another germ of a generic real analytic submanifold in $\mathbb{C}^{N}$, must be convergent.

ii) If $M$ is a connected generic holomorphically degenerate submanifold in $\mathbb{C}^{N}$, then, for any point $p \in M$, there exists a formal biholomorphism sending $(M, p)$ into itself which does not converge.

In view of these facts and other related results in the mapping problems ([2]), it appears likely that the subvariety $S$ in i) could be taken to be empty. In this paper, we actually prove such a result in the case of hypersurfaces. Our proof is based on an analysis of a so-called reflection function associated to the mapping and the hypersurfaces, which was used in many other situations $(c f .[11,13])$. We should also mention that, recently in [14], we showed that the real analytic subvariety $S$ in i) can also be taken to be empty when the generic submanifold $M$ is assumed to be real algebraic (and with no such assumption on the target manifold).

\section{Statement of main results}

Let $\left(M^{\prime}, p^{\prime}\right) \subset \mathbb{C}^{n}, n \geq 2$, be a germ at $p^{\prime}$ of a smooth real real-analytic hypersurface. Let $\rho^{\prime}=\rho^{\prime}(\zeta, \bar{\zeta})$ be a real analytic defining function for $M^{\prime}$ near $p^{\prime}$, i.e.,

$$
M^{\prime}=\left\{\zeta \in\left(\mathbb{C}^{n}, p^{\prime}\right): \rho^{\prime}(\zeta, \bar{\zeta})=0\right\} .
$$

After complexification of $\rho^{\prime}$, one defines the so-called invariant Segre varieties attached to $M^{\prime}$ by

$$
Q_{\omega}^{\prime}=\left\{\zeta \in\left(\mathbb{C}^{n}, p^{\prime}\right): \rho^{\prime}(\zeta, \bar{\omega})=0\right\},
$$

for $\omega$ close to $p^{\prime}$. We can assume, without loss of generality, that $p^{\prime}=0$ and $\frac{\partial \rho^{\prime}}{\partial \zeta_{n}}(0) \neq 0, \zeta=\left(\zeta^{\prime}, \zeta_{n}\right) \in \mathbb{C}^{n-1} \times \mathbb{C}$. Thus, the implicit function theorem exhibits any Segre variety as a graph of the form

$$
Q_{\omega}^{\prime}=\left\{\zeta \in\left(\mathbb{C}^{n}, 0\right): \zeta_{n}=\Phi^{\prime}\left(\bar{\omega}, \zeta^{\prime}\right)\right\}
$$

where $\Phi^{\prime}$ is a holomorphic function in its arguments in a neighborhood of $0 \in$ $\mathbb{C}^{2 n-1}$ and such that $\Phi^{\prime}(0)=0$. Equivalently, this Segre variety can be defined 
by

$$
Q_{\omega}^{\prime}=\left\{\zeta \in\left(\mathbb{C}^{n}, 0\right): \bar{\zeta}_{n}=\bar{\Phi}^{\prime}\left(\omega, \bar{\zeta}^{\prime}\right)\right\}
$$

Here, we have used the following notation. If $g=g(x)$ is some formal holomorphic power series in $\mathbb{C}[[x]], x=\left(x_{1}, \ldots, x_{k}\right), \bar{g}$ is the formal holomorphic power series obtained by taking the complex conjugates of the coefficients of $g$. (This convention of notation will be used throughout the paper.) Our main result is the following.

Theorem 2.1. Let $f:(M, 0) \rightarrow\left(M^{\prime}, 0\right)$ be a formal biholomorphism between two germs at 0 of real-analytic hypersurfaces in $\mathbb{C}^{n}$. Assume, furthermore, that $M$ is minimal at 0 . Then, the formal holomorphic map

$$
\mathbb{C}^{n} \times \mathbb{C}^{n-1} \ni(z, \lambda) \mapsto \bar{\Phi}^{\prime}(f(z), \lambda)
$$

is convergent.

Such a result has several applications. One of its main applications lies in the following theorem mentioned in the introduction.

Theorem 2.2. Any formal biholomorphic mapping between germs of minimal, holomorphically nondegenerate, real analytic hypersurfaces in $\mathbb{C}^{n}$ is convergent.

As explained in the introduction, the interest in such a result lies in the fact that, in view of [3], the condition of holomorphic nondegeneracy is optimal for the class of formal biholomorphisms. However, it remains an open problem to decide whether or not the condition of minimality is necessary in Theorem 2.2. Another application of Theorem 2.1 deals with partial convergence of formal biholomorphisms. For this, we refer the reader to $\S 7$.

Remark 1. After this work was completed, I received a preprint by J. Merker, "Convergence of formal biholomorphic mappings between minimal holomorphically nondegenerate real analytic hypersurfaces", in which a similar statement to Theorem 2.2 is given. In that preprint, Theorem 2.1 above is also stated for the special case where $M^{\prime}$ is a rigid and polynomial hypersurface.

\section{Definitions and notations}

3.1. Real analytic hypersurfaces. Let $M$ be a smooth real real-analytic hypersurface in $\mathbb{C}^{n}, n \geq 2$. Since the situation is purely local, we shall always work near a point $p \in M$, which will be assumed, without loss of generality, to be the origin. Let $\rho$ be a real analytic defining function for $M$ near 0 i.e.,

$$
M=\left\{z \in\left(\mathbb{C}^{n}, 0\right): \rho(z, \bar{z})=0\right\},
$$

with $d \rho \neq 0$ on $M$. The complexification $\mathcal{M}$ of $M$ is the complex hypersurface through 0 in $\mathbb{C}^{2 n}$ given by

$$
\mathcal{M}=\left\{(z, w) \in\left(\mathbb{C}^{2 n}, 0\right): \rho(z, w)=0\right\} .
$$


We shall assume, without loss of generality, the coordinates $z=\left(z^{\prime}, z_{n}\right) \in \mathbb{C}^{n}$ are chosen so that $\frac{\partial \rho}{\partial z_{n}}(0) \neq 0$. In this case, we define the following holomorphic vector fields tangent to $\mathcal{M}$

$$
\mathcal{L}_{j}=\frac{\partial \rho}{\partial w_{n}}(z, w) \frac{\partial}{\partial w_{j}}-\frac{\partial \rho}{\partial w_{j}}(z, w) \frac{\partial}{\partial w_{n}}, j=1, \ldots, n-1,
$$

which are the complexifications of the usual $(0,1)$ vector fields tangent to $M$. We recall that for a point $w$ near 0, its associated Segre surface is the complex hypersurface defined by $Q_{w}=\left\{z \in\left(\mathbb{C}^{n}, 0\right): \rho(z, \bar{w})=0\right\}$. Observe that by the complex analytic implicit function theorem, each Segre variety can be described as a graph of the form

$$
Q_{w}=\left\{z \in\left(\mathbb{C}^{n}, 0\right): z_{n}=\Phi\left(\bar{w}, z^{\prime}\right)\right\}
$$

$\Phi$ denoting a convergent power series in some neighborhood of the origin in $\mathbb{C}^{2 n-1}$ satisfying the relations $\Phi(0)=0$ and

$$
\Phi\left(w^{\prime}, \bar{\Phi}\left(z, w^{\prime}\right), z^{\prime}\right) \equiv z_{n}, \quad\left(z, w^{\prime}\right) \in \mathbb{C}^{n} \times \mathbb{C}^{n-1} .
$$

Equation (3.2) is a consequence of the fact that $M$ is a real hypersurface. The coordinates $z$ are said to be normal with respect to $M$ if the additional condition

$$
\Phi\left(w^{\prime}, w_{n}, 0\right)=\Phi\left(0, w_{n}, z^{\prime}\right) \equiv w_{n}, \quad\left(w^{\prime}, w_{n}, z^{\prime}\right) \in \mathbb{C}^{n-1} \times \mathbb{C} \times \mathbb{C}^{n-1},
$$

holds. It is well-known that given a real-analytic hypersurface through the origin, one can always construct such coordinates [9]. Thus, from now on and for simplicity, we will always assume that the $z$-coordinates are chosen to be normal for the manifold $M$.

The real analytic hypersurface $M$ is called minimal at 0 (in the sense of Trépreau and Tumanov), or, equivalently of finite type (in the sense of Kohn and Bloom-Graham) if it does not contain any complex-analytic hypersurface through 0. To use such a nondegeneracy condition, we will need the Segre set mappings associated to $M$ (see [2]) up to order 3, which, in normal coordinates, are the following three maps :

$$
\begin{aligned}
\left(\mathbb{C}^{n-1}, 0\right) \ni z^{\prime} \mapsto v_{1}\left(z^{\prime}\right) & =\left(z^{\prime}, 0\right) \in \mathbb{C}^{n}, \\
\left(\mathbb{C}^{2 n-2}, 0\right) \ni\left(z^{\prime}, \xi\right) \mapsto v_{2}\left(z^{\prime}, \xi\right) & =\left(z^{\prime}, \Phi\left(\xi, 0, z^{\prime}\right)\right) \in \mathbb{C}^{n}, \\
\left(\mathbb{C}^{3 n-3}, 0\right) \ni\left(z^{\prime}, \xi, \eta\right) \mapsto v_{3}\left(z^{\prime}, \xi, \eta\right) & =\left(z^{\prime}, \Phi\left(\xi, \bar{\Phi}(\eta, 0, \xi), z^{\prime}\right)\right) \in \mathbb{C}^{n} .
\end{aligned}
$$

These maps are of fundamental importance since they parametrize the so-called Segre sets (up to order three) associated to $M$. Moreover, the interest in such Segre sets or Segre set mappings lies in the fact that the minimality assumption is equivalent to the fact that the generic rank of $v_{2}$ (and also $v_{3}$ ) equals $n$ (see [2]). This will be useful for the proof of Theorem 2.1.

All the notations introduced in this section will be used for the source hypersurface $M$. For the target real analytic hypersurface $M^{\prime}$, we shall use the notations introduced in $\S 2$, before the statement of Theorem 2.1. In particular, 
we denote by $z$ the coordinates in the source space and by $\zeta$ the coordinates at the target space.

3.2. Some commutative algebra. We recall here, for the reader's convenience, some basic definitions, needed in $\S 7$, about regular local rings and their ideals. All these definitions can be found for instance in $[12,17]$.

Let $A$ be a Noetherian ring. If $I$ and $J$ are two ideals of $A$, we use the notation $I<J$ to mean that $I \subset J$ and $I \neq J$. Given a prime ideal $I \subseteq A$, the height of $I$ is defined by the formula

$$
h(I)=\max \left\{k \in \mathbb{N}:\{0\}<I_{1}<\ldots<I_{k}=I\right\},
$$

where $I_{1}, \ldots, I_{k}$ are prime ideals of $A$. If $J$ is any ideal of $A$, we define the height of $J$ by the formula

$$
h(J)=\inf \{h(I): J \subset I, I \text { prime ideal of } A\} .
$$

If $A$ is furthermore assumed to be a local ring, one defines the Krull dimension of $A$ to be the height of its maximal ideal. Observe that if $A$ is a Noetherian local ring and if $I$ is a proper ideal of $A$, the quotient ring $A / I$ is also a Noetherian local ring. This allows one to consider the Krull dimension of such a ring. Finally, a Noetherian local ring is said to be regular if its maximal ideal has $\delta$ generators, where $\delta$ is the Krull dimension of the ring $A$. The rings of formal holomorphic power series or convergent power series in $p$ indeterminates, $p \in \mathbb{N}^{*}$, are regular rings of Krull dimension $p$ [17].

\section{Two convergence results}

In this section, we first state and prove a convenient lemma which will be used twice in the paper. This lemma may already be known.

Lemma 4.1. Let $\left(u_{i}(t)\right)_{i \in I}$ be a family of convergent power series in $\mathbb{C}\{t\}, t=$ $\left(t_{1}, \ldots, t_{q}\right), q \in \mathbb{N}^{*}$. Let also $\left(\mathcal{K}_{i}(\varsigma)\right)_{i \in I}$ be a family of convergent power series in $\mathbb{C}\{\varsigma\}, \varsigma=\left(\varsigma_{1}, \ldots, \varsigma_{r}\right), r \in \mathbb{N}^{*}$. Assume that:

(i) There exists $R>0$ such that the radius of convergence of any $\mathcal{K}_{i}, i \in I$, is at least $R$.

(ii) For all $\varsigma \in \mathbb{C}^{r}$ with $|\varsigma|<R,\left|\mathcal{K}_{i}(\varsigma)\right| \leq C_{i}$, with $C_{i}>0$.

(iii) There exists $V(t)=\left(V_{1}(t), \ldots, V_{r}(t)\right) \in(\mathbb{C}[[t]])^{r}, V(0)=0$, such that $\left(\mathcal{K}_{i} \circ V\right)(t)=u_{i}(t)$ (in $\left.\mathbb{C}[[t]]\right)$ for all $i \in I$.

Then, there exists $R^{\prime}>0$ such that the radius of convergence of any $u_{i}, i \in I$, is at least $R^{\prime}$ and such that for all $t \in \mathbb{C}^{q}$ with $|t|<R^{\prime},\left|u_{i}(t)\right| \leq C_{i}$.

Proof. By Artin's approximation theorem [1], one can find a convergent power series mapping $\vartheta(t)=\left(\vartheta_{1}(t), \ldots, \vartheta_{r}(t)\right) \in(\mathbb{C}\{t\})^{r}$ such that $\vartheta(0)=0$ and for all $i \in I,\left(\mathcal{K}_{i} \circ \vartheta\right)(t)=u_{i}(t)$ in $\mathbb{C}\{t\}$. Let $R^{\prime}>0$ so that if $|t|<R^{\prime}$ then $|\vartheta(t)|<R$. Since the radius of convergence of the $\mathcal{K}_{i}, i \in I$, is at least $R$, the radius of convergence of any $\mathcal{K}_{i} \circ \vartheta, i \in I$, is at least $R^{\prime}$. Thus, the family $\left(u_{i}(t)\right)_{i \in I}$ has a radius of convergence at least equal to $R^{\prime}$ and for all $t \in \mathbb{C}^{q}$ with $|t|<R^{\prime}$, one has $\left|u_{i}(t)\right|=\left|\left(\mathcal{K}_{i} \circ \vartheta\right)(t)\right| \leq C_{i}, i \in I$. 
To derive Theorem 2.2 from Theorem 2.1, we will need the following consequence of Artin's approximation theorem, which is contained for instance in [15]. (See also $\S 7$ for another formulation of such a result.)

Proposition 4.2. Let $R(x, y)=\left(R_{1}(x, y), \ldots, R_{r}(x, y)\right) \in(\mathbb{C}\{x, y\})^{r}, x \in \mathbb{C}^{q}$, $y \in \mathbb{C}^{r}, q, r \in \mathbb{N}^{*}$. Let $g(x)=\left(g_{1}(x), \ldots, g_{r}(x)\right) \in(\mathbb{C}[[x]])^{r}$ satisfy $R(x, g(x))=$ 0. If $\operatorname{det}\left(\frac{\partial R}{\partial y}(x, g(x))\right) \not \equiv 0$ in $\mathbb{C}[[x]]$, then $g(x)$ is convergent.

Proof. We reproduce here the arguments of [15]. Write

$$
R(x, y)-R(x, z)=Q(x, y, z) \cdot(y-z)
$$

where $Q$ is an $r \times r$ complex-analytic matrix such that $Q(x, y, y)=\frac{\partial R}{\partial y}(x, y)$; i.e., $Q(x, y, z)=\int_{0}^{1} \frac{\partial R}{\partial y}(x, t y+(1-t) z) d t$. By assumption, we know that we have $\operatorname{det} Q(x, g(x), g(x)) \not \equiv 0$. This implies that one can find an integer $k_{g}$ such that if $H(x)$ is any formal power series which agrees up to order $k_{g}$ with $g$ then $\operatorname{det} Q(x, g(x), H(x)) \not \equiv 0$. For this integer $k_{g}$, according to Artin's approximation theorem, one can find a convergent power series $H_{0}(x)$ satisfying $R\left(x, H_{0}(x)\right)=0$ and agreeing with $g(x)$ up to order $k_{g}$. By (4.1), we get $Q\left(x, g(x), H_{0}(x)\right) \cdot(g(x)-$ $\left.H_{0}(x)\right) \equiv 0$ in $\mathbb{C}[[x]]$. Since $\operatorname{det} Q\left(x, g(x), H_{0}(x)\right) \not \equiv 0$, we obtain $g(x)=H_{0}(x)$ and thus $g$ is convergent.

\section{The reflection principle}

Let $f:(M, 0) \rightarrow\left(M^{\prime}, 0\right)$ be a formal biholomorphic mapping between germs at 0 of real analytic hypersurfaces in $\mathbb{C}^{n}$. We shall use the notations introduced in $\S 2$ and $\S 3$. In particular, we denote $f=f(z)=\left(f_{1}(z), \ldots, f_{n}(z)\right)=$ $\left(f^{\prime}(z), f_{n}(z)\right)$ in the $\zeta$-coordinates. In this section, we shall make no further assumptions on $M$ and $M^{\prime}$.

As in $[13,14]$, we define the following formal holomorphic power series

$$
\mathbb{C}^{n} \times \mathbb{C}^{n-1} \ni(z, \lambda) \mapsto \mathcal{R}(z, \lambda):=\bar{\Phi}^{\prime}(f(z), \lambda) .
$$

The goal of this section is to prove the following proposition.

Proposition 5.1. Let $f:(M, 0) \rightarrow\left(M^{\prime}, 0\right)$ be a formal biholomorphism between germs at 0 of real analytic hypersurfaces in $\mathbb{C}^{n}$, and $\mathcal{R}$ defined by (5.1). Then for any multi-index $\gamma \in \mathbb{N}^{n}$, the formal holomorphic map

$$
\left.\left(\mathbb{C}^{2 n-2}, 0\right) \ni\left(z^{\prime}, \lambda\right) \mapsto\left(\partial_{z}^{\gamma} \mathcal{R}(z, \lambda)\right)\right|_{z=v_{1}\left(z^{\prime}\right)}
$$

is convergent in some neighborhood $V_{\gamma}$ of $0 \in \mathbb{C}^{2 n-2}$. Here, $v_{1}$ is the first Segre set mapping for $M$ as defined in (3.3).

Before proceeding to the proof of Proposition 5.1, we need a preliminary lemma (Lemma 5.2 below). Since $f$ maps formally $M$ into $M^{\prime}$, there exists $a(z, \bar{z}) \in \mathbb{C}[[z, \bar{z}]]$ such that

$$
\overline{f_{n}(z)}-\bar{\Phi}^{\prime}\left(f(z), \overline{f^{\prime}(z)}\right)=a(z, \bar{z}) \rho(z, \bar{z}) \text {, in } \mathbb{C}[[z, \bar{z}]] \text {. }
$$


Equivalently, we have

$$
\bar{f}_{n}(w)-\bar{\Phi}^{\prime}\left(f(z), \bar{f}^{\prime}(w)\right)=a(z, w) \rho(z, w), \text { in } \mathbb{C}[[z, w]] .
$$

We write $\bar{\Phi}_{\lambda^{\alpha}}^{\prime}(\omega, \lambda)$ for $\partial_{\lambda}^{\alpha} \bar{\Phi}^{\prime}(\omega, \lambda)$. By applying the vector fields $\mathcal{L}_{j}, j=$ $1, \ldots, n-1$, as defined by (3.1), to (5.2) and using the fact that $f$ is invertible, one obtains the following known statement (see [4] for instance).

Lemma 5.2. Under the assumptions of Proposition 5.1, one has, for any multindex $\alpha \in \mathbb{N}^{n-1}$, the formal power series identity

$$
\bar{\Phi}_{\lambda^{\alpha}}^{\prime}\left(f(z), \bar{f}^{\prime}(w)\right)=\chi_{\alpha}\left(\left(\partial^{\beta} \bar{f}(w)\right)_{|\beta| \leq|\alpha|}, z, w\right),(z, w) \in \mathcal{M},
$$

where each $\chi_{\alpha}$ is a convergent power series of its arguments.

Proof of Proposition 5.1. We write the expansion

$$
\bar{\Phi}^{\prime}(\omega, \lambda)=\sum_{\alpha \in \mathbb{N}^{n-1}} \phi_{\alpha}^{\prime}(\omega) \lambda^{\alpha} .
$$

For the sake of clarity, we shall first give the proof of the Proposition in the case $\gamma=0$.

The CASE $\gamma=0$. We restrict all the identities given by Lemma 5.2 to the $(n-1)$-dimensional subspace

$$
\left\{\left(0, v_{1}\left(z^{\prime}\right)\right): z^{\prime} \in\left(\mathbb{C}^{n-1}, 0\right)\right\} \subset \mathcal{M} .
$$

This gives, for any multiindex $\alpha \in \mathbb{N}^{n-1}$,

$$
\alpha !\left(\phi_{\alpha}^{\prime} \circ f \circ v_{1}\right)\left(z^{\prime}\right)=\chi_{\alpha}\left(\left(\partial^{\beta} \bar{f}(0)\right)_{|\beta| \leq|\alpha|}, v_{1}\left(z^{\prime}\right), 0\right):=u_{\alpha}\left(z^{\prime}\right),
$$

where $\phi_{\alpha}^{\prime}$ is given by (5.3). Observe that for each multiindex $\alpha, u_{\alpha}\left(z^{\prime}\right)$ is convergent.

To show that $\mathbb{C}^{n-1} \times \mathbb{C}^{n-1} \ni\left(z^{\prime}, \lambda\right) \mapsto \mathcal{R}\left(v_{1}\left(z^{\prime}\right), \lambda\right)$ is convergent, we claim that it suffices to show that there exists $a>0$ and $R_{0}>0$ such that the radius of convergence of each $u_{\alpha}$ is at least $a$, and such that the following Cauchy estimates hold:

$$
\forall \alpha \in \mathbb{N}^{n-1}, \forall z^{\prime} \in \mathbb{C}^{n-1},\left|z^{\prime}\right|<a,\left|u_{\alpha}\left(z^{\prime}\right)\right| \leq \alpha ! R_{0}^{|\alpha|+1} .
$$

Indeed, if (5.5) holds, then the formal holomorphic power series

$$
\mathbb{C}^{n-1} \times \mathbb{C}^{n-1} \ni\left(z^{\prime}, \lambda\right) \mapsto \mathcal{R}_{0}\left(z^{\prime}, \lambda\right):=\sum_{|\alpha|=0}^{\infty} \frac{u_{\alpha}\left(z^{\prime}\right)}{\alpha !} \lambda^{\alpha}
$$

defines a convergent power series in $B_{n-1}(0, a) \times B_{n-1}\left(0,1 / 2 R_{0}\right)$. (Here and in what follows, for any $c>0$ and for any $k \in \mathbb{N}^{*}, B_{k}(0, c)$ denotes the euclidean ball centered at 0 in $\mathbb{C}^{k}$ of radius $c$.) Moreover, by (5.4) and (5.1), we have for any multiindex $\alpha \in \mathbb{N}^{n-1}$,

$$
\left[\frac{\partial^{|\alpha|} \mathcal{R}_{0}}{\partial \lambda^{\alpha}}\left(z^{\prime}, \lambda\right)\right]_{\lambda=0}=\left[\frac{\partial^{|\alpha|} \mathcal{R}}{\partial \lambda^{\alpha}}\left(v_{1}\left(z^{\prime}\right), \lambda\right)\right]_{\lambda=0}
$$

in $\mathbb{C}\left[\left[z^{\prime}\right]\right]$ and hence

$$
\mathcal{R}_{0}\left(z^{\prime}, \lambda\right)=\mathcal{R}\left(v_{1}\left(z^{\prime}\right), \lambda\right)
$$


This proves that under the assumption (5.5), $\mathcal{R}\left(v_{1}\left(z^{\prime}\right), \lambda\right)$ is a convergent power series in $\left(z^{\prime}, \lambda\right)$. It remains to prove (5.5). Since $\bar{\Phi}^{\prime}$ is holomorphic in a neighborhood of $0 \in \mathbb{C}^{2 n-1}$, in view of (5.3), one can find $\delta>0$ and a constant $R>0$ such that for any multiindices $\alpha \in \mathbb{N}^{n-1}, \nu \in \mathbb{N}^{n}$,

$$
\forall \omega \in \mathbb{C}^{n},|\omega|<\delta,\left|\frac{\partial^{|\nu|} \phi_{\alpha}^{\prime}(\omega)}{\partial \omega^{\nu}}\right| \leq \nu ! R^{|\alpha|+|\nu|+1} .
$$

In view of (5.4) and (5.7) (in the case $\nu=0$ ), we can apply Lemma 4.1 to conclude that there exists $a>0$ such that the family $\left(u_{\alpha}\left(z^{\prime}\right)\right)_{\alpha \in \mathbb{N}^{n-1}}$ is convergent in $B_{n-1}(0, a)$ and such that $(5.5)$ holds with $R_{0}=R$. This finishes the proof of Proposition 5.1 in the case $\gamma=0$.

THE CASE $|\gamma|>0$. We proceed now to the proof of Proposition 5.1 for general $\gamma \in \mathbb{N}^{n}$. For this, we need the following lemma.

Lemma 5.3. Under the assumptions of Proposition 5.1, for any multiindices $\alpha \in \mathbb{N}^{n-1}, \gamma \in \mathbb{N}^{n}$, the formal holomorphic power series

$$
\left.\mathbb{C}^{n-1} \ni z^{\prime} \mapsto \partial_{z}^{\gamma}\left(\left(\phi_{\alpha}^{\prime} \circ f\right)(z)\right)\right|_{z=v_{1}\left(z^{\prime}\right)}
$$

is convergent.

Proof of Lemma 5.3. We prove the Lemma by induction on $|\gamma|$ (for any multiindex $\alpha \in \mathbb{N}^{n-1}$ ). For $\gamma=0$, the statement follows from (5.4), as we previously noticed. Let $\gamma \in \mathbb{N}^{n}$. For $\alpha \in \mathbb{N}^{n-1}$, using Lemma 5.2, we obtain for $\left(z^{\prime}, z_{n}, 0, z_{n}\right) \in(\mathcal{M}, 0)$,

$$
\bar{\Phi}_{\lambda^{\alpha}}^{\prime}\left(f(z), \bar{f}^{\prime}\left(0, z_{n}\right)\right)=\chi_{\alpha}\left(\left(\partial^{\beta} \bar{f}\left(0, z_{n}\right)\right)_{|\beta| \leq|\alpha|}, z, 0, z_{n}\right) .
$$

If we apply $\partial_{z}^{\gamma}$ to this equation, we obtain

$$
\frac{\partial^{|\gamma|}}{\partial z^{\gamma}}\left[\mathcal{R}_{\lambda^{\alpha}}\left(z, \bar{f}^{\prime}\left(0, z_{n}\right)\right)\right]=\frac{\partial^{|\gamma|}}{\partial z^{\gamma}}\left[\chi_{\alpha}\left(\left(\partial^{\beta} \bar{f}\left(0, z_{n}\right)\right)_{|\beta| \leq|\alpha|}, z, 0, z_{n}\right)\right] .
$$

One can easily check that this implies that there exist a polynomial $\mathcal{S}_{\gamma}$ such that the left-hand side of (5.8) is equal to

$$
\begin{aligned}
& \mathcal{R}_{z^{\gamma} \lambda^{\alpha}}\left(z, \bar{f}^{\prime}\left(0, z_{n}\right)\right)+ \\
& \mathcal{S}_{\gamma}\left[\left(\mathcal{R}_{z^{\nu} \lambda^{\beta}}\left(z, \bar{f}^{\prime}\left(0, z_{n}\right)\right)\right)_{\substack{|\nu|<|<|<|| \beta|\leq| \alpha|+| \gamma \mid}},\left(\partial^{\mu} \bar{f}\left(0, z_{n}\right)\right)_{|\mu| \leq|\gamma|}\right],
\end{aligned}
$$

where $\mu, \nu \in \mathbb{N}^{n}, \beta \in \mathbb{N}^{n-1}$. Furthermore, we observe that the right-hand side of (5.8) can be written in the form

$$
\chi_{\alpha, \gamma}^{1}\left(\left(\partial^{\beta} \bar{f}\left(0, z_{n}\right)\right)_{|\beta| \leq|\alpha|+|\gamma|}, z\right),
$$


where $\chi_{\alpha, \gamma}^{1}$ is a convergent power series. Restricting (5.8), (5.9) and (5.10) to $z=v_{1}\left(z^{\prime}\right)$, one obtains

$$
\begin{aligned}
& \left.\alpha ! \partial_{z}^{\gamma}\left(\left(\phi_{\alpha}^{\prime} \circ f\right)(z)\right)\right|_{z=v_{1}\left(z^{\prime}\right)}+ \\
& \mathcal{S}_{\gamma}\left[\left(\left.\beta ! \partial_{z}^{\nu}\left(\left(\phi_{\beta}^{\prime} \circ f\right)(z)\right)\right|_{z=v_{1}\left(z^{\prime}\right)}\right)_{\substack{|\beta| \leq|\alpha| \gamma|+| \gamma|| \nu \mid}},\left(\partial^{\mu} \bar{f}(0)\right)_{|\mu| \leq|\gamma|}\right]= \\
& \chi_{\alpha, \gamma}^{1}\left(\left(\partial^{\beta} \bar{f}(0)\right)_{|\beta| \leq|\alpha|+|\gamma|}, z^{\prime}, 0\right) .
\end{aligned}
$$

The induction hypothesis tells us that for any multiindex $\beta \in \mathbb{N}^{n-1}$ and for any multiindex $\nu \in \mathbb{N}^{n}$ such that $|\nu|<|\gamma|$, the formal holomorphic power series

$$
\left.z^{\prime} \in \mathbb{C}^{n-1} \mapsto \partial_{z}^{\nu}\left(\left(\phi_{\beta}^{\prime} \circ f\right)(z)\right)\right|_{z=v_{1}\left(z^{\prime}\right)}
$$

is convergent. Thus, we obtain the desired similar statement for $\mathbb{C}^{n-1} \ni z^{\prime} \mapsto$ $\left.\partial_{z}^{\gamma}\left(\left(\phi_{\alpha}^{\prime} \circ f\right)(z)\right)\right|_{z=v_{1}\left(z^{\prime}\right)}$, for any multiindex $\alpha \in \mathbb{N}^{n-1}$.

We come back to the proof of Proposition 5.1. For all multiindices $\gamma \in \mathbb{N}^{n}$, $\alpha \in \mathbb{N}^{n-1}$, we put

$$
\Psi_{\alpha, \gamma}\left(z^{\prime}\right):=\left.\alpha ! \partial_{z}^{\gamma}\left(\left(\phi_{\alpha}^{\prime} \circ f\right)(z)\right)\right|_{z=v_{1}\left(z^{\prime}\right)}=\mathcal{R}_{z^{\gamma} \lambda^{\alpha}}\left(v_{1}\left(z^{\prime}\right), 0\right) .
$$

By Lemma 5.3, the $\Psi_{\alpha, \gamma}\left(z^{\prime}\right)$ are convergent. We now fix $\gamma,|\gamma| \geq 1$. We want to prove that $\mathcal{R}_{z^{\gamma}}\left(v_{1}\left(z^{\prime}\right), \lambda\right)$ is convergent in some neighborhood $V_{\gamma}$ of $0 \in \mathbb{C}^{2 n-2}$. For this, as in the case $\gamma=0$, it suffices to prove that one can find $q_{\gamma}>0$ and $R_{\gamma}>0$ such that the radius of convergence of the family $\left(\Psi_{\alpha, \gamma}\right)_{\alpha \in \mathbb{N}^{n-1}}$ is at least $q_{\gamma}$ and such that the following estimates hold:

$$
\forall \alpha \in \mathbb{N}^{n-1}, \forall z^{\prime} \in \mathbb{C}^{n-1},\left|z^{\prime}\right|<q_{\gamma},\left|\Psi_{\alpha, \gamma}\left(z^{\prime}\right)\right| \leq \alpha ! R_{\gamma}^{|\alpha|+1} .
$$

We first observe that for any multiindex $\nu \in \mathbb{N}^{n}$ with $|\nu| \leq|\gamma|$, there exists a universal polynomial $\mathcal{P}_{\nu, \gamma}$, such that

$$
\begin{aligned}
\mathcal{R}_{z^{\gamma} \lambda^{\alpha}}(z, \lambda)=\partial_{z}^{\gamma}\left(\bar{\Phi}_{\lambda^{\alpha}}^{\prime}(f(z), \lambda)\right)= & \\
& \sum_{|\nu| \leq|\gamma|} \mathcal{P}_{\nu, \gamma}\left(\left(\partial^{\beta} f(z)\right)_{1 \leq|\beta| \leq|\gamma|}\right) \bar{\Phi}_{\omega^{\nu} \lambda^{\alpha}}^{\prime}(f(z), \lambda) .
\end{aligned}
$$

This means in particular that the polynomials $\mathcal{P}_{\nu, \gamma},|\nu| \leq|\gamma|$, are independent of $\alpha$. Putting $\lambda=0$ and $z=v_{1}\left(z^{\prime}\right)$ in (5.14), we obtain

$$
\begin{aligned}
& \mathcal{R}_{z^{\gamma} \lambda^{\alpha}}\left(v_{1}\left(z^{\prime}\right), 0\right)= \\
& \alpha ! \sum_{|\nu| \leq|\gamma|} \mathcal{P}_{\nu, \gamma}\left(\left(\left(\partial^{\beta} f\right)\left(v_{1}\left(z^{\prime}\right)\right)\right)_{1 \leq|\beta| \leq|\gamma|}\right)\left(\frac{\partial^{|\nu|} \phi_{\alpha}^{\prime}}{\partial \omega^{\nu}} \circ f \circ v_{1}\right)\left(z^{\prime}\right) .
\end{aligned}
$$

Recall that $\gamma$ is fixed. For $\alpha \in \mathbb{N}^{n-1}$, consider the convergent power series of the variables $\left(\left(\Lambda_{\beta}\right)_{1 \leq|\beta| \leq|\gamma|}, \omega\right)$ defined by

$$
h_{\alpha, \gamma}\left(\left(\Lambda_{\beta}\right)_{1 \leq|\beta| \leq|\gamma|}, \omega\right):=\alpha ! \sum_{|\nu| \leq|\gamma|} \mathcal{P}_{\nu, \gamma}\left(\left(\Lambda_{\beta}+\partial^{\beta} f(0)\right)_{1 \leq|\beta| \leq|\gamma|}\right) \frac{\partial^{|\nu|} \phi_{\alpha}^{\prime}}{\partial \omega^{\nu}}(\omega) .
$$


Let $r(\gamma)=n \operatorname{Card}\left\{\nu \in \mathbb{N}^{n}: 1 \leq|\nu| \leq|\gamma|\right\}$. In view of (5.7), each $h_{\alpha, \gamma}$, $\alpha \in \mathbb{N}^{n-1}$, is convergent in $B_{r(\gamma)}(0,1) \times B_{n-1}(0, \delta)$. Moreover, we have the following estimates for $\left(\left(\Lambda_{\beta}\right)_{1 \leq|\beta| \leq|\gamma|}, \omega\right) \in B_{r(\gamma)}(0,1) \times B_{n-1}(0, \delta)$,

$$
\left|h_{\alpha, \gamma}\left(\left(\Lambda_{\beta}\right)_{1 \leq|\beta| \leq|\gamma|}, \omega\right)\right| \leq \alpha ! \sum_{|\nu| \leq|\gamma|}\left|\mathcal{P}_{\nu, \gamma}\left(\left(\Lambda_{\beta}+\partial^{\beta} f(0)\right)_{1 \leq|\beta| \leq|\gamma|}\right)\right| \nu ! R^{|\alpha|+|\nu|+1} .
$$

Put

$$
\begin{aligned}
& C_{\gamma}:=\sup \left\{\left|\mathcal{P}_{\nu, \gamma}\left(\left(\Lambda_{\beta}+\partial^{\beta} f(0)\right)_{1 \leq|\beta| \leq|\gamma|}\right)\right|:\right. \\
&\left.|\nu| \leq|\gamma|, \quad\left(\Lambda_{\beta}^{n}\right)_{1 \leq|\beta| \leq|\gamma|} \in B_{r(\gamma)}(0,1)\right\}
\end{aligned}
$$

This implies that in $B_{r(\gamma)}(0,1) \times B_{n-1}(0, \delta)$, the following estimates hold for some suitable constant $C_{\gamma}^{1}$ :

$$
\left|h_{\alpha, \gamma}\left(\left(\Lambda_{\beta}\right)_{1 \leq|\beta| \leq|\gamma|}, \omega\right)\right| \leq \alpha ! C_{\gamma} \sum_{|\nu| \leq|\gamma|} \nu ! R^{|\alpha|+|\nu|+1} \leq C_{\gamma}^{1} \alpha ! R^{|\alpha|+|\gamma|+1} .
$$

From this, we see that there exists $R_{\gamma}>0$ such that for $\left(\left(\Lambda_{\beta}\right)_{1 \leq|\beta| \leq|\gamma|}, \omega\right) \in$ $B_{r(\gamma)}(0,1) \times B_{n-1}(0, \delta)$,

$$
\left|h_{\alpha, \gamma}\left(\left(\Lambda_{\beta}\right)_{1 \leq|\beta| \leq|\gamma|}, \omega\right)\right| \leq \alpha ! R_{\gamma}^{|\alpha|+1} .
$$

In view of (5.12) and (5.15), we have for any multiindex $\alpha \in \mathbb{N}^{n-1}$,

$$
h_{\alpha, \gamma}\left(\left(\left(\partial^{\beta} f\right)\left(v_{1}\left(z^{\prime}\right)\right)-\partial^{\beta} f(0)\right)_{1 \leq|\beta| \leq|\gamma|},\left(f \circ v_{1}\right)\left(z^{\prime}\right)\right)=\Psi_{\alpha, \gamma}\left(z^{\prime}\right),
$$

as formal power series in $z^{\prime}$. Thus, in view of (5.17), we are in a position to apply Lemma 4.1 to conclude that there exists $q_{\gamma}>0$ such that the family $\left(\Psi_{\alpha, \gamma}\left(z^{\prime}\right)\right)_{\alpha \in \mathbb{N}^{n-1}}$ is convergent in $B_{n-1}\left(0, q_{\gamma}\right)$, in which, moreover, the desired estimates (5.13) hold. This implies that $\mathcal{R}_{z^{\gamma}}\left(v_{1}\left(z^{\prime}\right), \lambda\right) \in \mathbb{C}\left\{z^{\prime}, \lambda\right\}$. The proof of Proposition 5.1 is thus complete.

Remark 2. It is clear that Proposition 5.1 still holds in higher codimension with the same proof. More precisely, the following holds. Let $M, M^{\prime}$ be two germs through the origin in $\mathbb{C}^{n}, n \geq 2$, of smooth real real-analytic generic submanifolds of CR dimension $N$ and of real codimension $d$. Let $f:(M, 0) \rightarrow\left(M^{\prime}, 0\right)$ be a formal biholomorphic map. Assume that the coordinates at the target space $\zeta=\left(\zeta^{\prime}, \zeta^{*}\right) \in \mathbb{C}^{N} \times \mathbb{C}^{d}$ are chosen so that, near the origin, $M^{\prime}=$ $\left\{\left(\zeta^{\prime}, \zeta^{*}\right) \in\left(\mathbb{C}^{n}, 0\right): \bar{\zeta}^{*}=\bar{\Phi}^{\prime}\left(\zeta, \bar{\zeta}^{\prime}\right)\right\}$, for some $\mathbb{C}^{d}$-valued holomorphic map $\bar{\Phi}^{\prime}=\left(\bar{\Phi}_{1}^{\prime}, \ldots, \bar{\Phi}_{d}^{\prime}\right)$ near $0 \in \mathbb{C}^{2 N+d}$. Assume also that the coordinates at the source space are chosen to be normal coordinates for $M$. Then, if we define $\mathbb{C}^{n} \times \mathbb{C}^{N} \ni(z, \lambda) \mapsto \mathcal{R}(z, \lambda):=\bar{\Phi}^{\prime}(f(z), \lambda) \in \mathbb{C}^{d}$, the following holds. For any multiindex $\gamma \in \mathbb{N}^{n}$, the formal holomorphic power series mapping

$$
\mathbb{C}^{N} \times \mathbb{C}^{N} \ni\left(z^{\prime}, \lambda\right) \mapsto \mathcal{R}_{z^{\gamma}}\left(\left(z^{\prime}, 0\right), \lambda\right) \in \mathbb{C}^{d}
$$

is convergent. 


\section{Proofs of Theorem 2.1 and Theorem 2.2}

For the proof of Theorem 2.1, we first need to prove a lemma (also used in [14]) which will allow us to bypass the second Segre set and to work directly on the third Segre set.

Lemma 6.1. Let $\mathcal{T}(x, u)=\left(\mathcal{T}_{1}(x, u), \ldots, \mathcal{T}_{r}(x, u)\right) \in(\mathbb{C}[[x, u]])^{r}, x \in \mathbb{C}^{q}$, $u \in \mathbb{C}^{s}$, with $\mathcal{T}(0)=0$. Assume that $\mathcal{T}(x, u)$ satisfies an identity in the ring $\mathbb{C}[[x, u, y]], y \in \mathbb{C}^{q}$, of the form

$$
\varphi(\mathcal{T}(x, u) ; x, u, y)=0
$$

where $\varphi \in \mathbb{C}[[W, x, u, y]]$ with $W \in \mathbb{C}^{r}$. Assume, furthermore, that for any multi-index $\beta \in \mathbb{N}^{q}$, the formal power series $\left[\frac{\partial^{|\beta|} \varphi}{\partial y^{\beta}}(W ; x, u, y)\right]_{y=x}$ is convergent, i.e., belongs to $\mathbb{C}\{W, x, u\}$. Then, for any given positive integer e, there exists an r-tuple of convergent power series $\mathcal{T}^{e}(x, u) \in(\mathbb{C}\{x, u\})^{r}$ such that $\varphi\left(\mathcal{T}^{e}(x, u) ; x, u, y\right)=0$ in $\mathbb{C}[[x, u, y]]$ and such that $\mathcal{T}^{e}(x, u)$ agrees up to order $e$ (at 0) with $\mathcal{T}(x, u)$.

Proof. First observe that $\mathcal{T}(x, u)$ is a formal power series solution of the analytic system in the unknown $W$,

$$
\left[\frac{\partial^{|\beta|} \varphi}{\partial y^{\beta}}(W ; x, u, y)\right]_{y=x} \equiv 0, \quad \beta \in \mathbb{N}^{q} .
$$

Thus, an application of Artin's approximation theorem [1] gives, for any positive integer $e$, an $r$-tuple of convergent power series $\mathcal{T}^{e}(x, u) \in(\mathbb{C}\{x, u\})^{r}$ solution in $W$ of $(6.1)$, and which agrees up to order $e$ with $\mathcal{T}(x, u)$. The Lemma follows by noticing that $\varphi\left(\mathcal{T}^{e}(x, u) ; x, u, y\right) \equiv 0$ in $\mathbb{C}[[x, u, y]]$ if and only if $\mathcal{T}^{e}(x, u)$ is solution of (6.1). The proof of Lemma 6.1 is complete.

The following proposition will also be useful in the proof of Theorem 2.1 (see [8, Lemma 7.8] for a proof for instance).

Proposition 6.2. Let $\mathcal{J}(x)=\left(\mathcal{J}_{1}(x), \ldots, \mathcal{J}_{r}(x)\right) \in(\mathbb{C}\{x\})^{r}, x \in \mathbb{C}^{k}, k, r \geq 1$, $\mathcal{J}(0)=0$, and $\mathcal{V}(t) \in \mathbb{C}[[t]], t \in \mathbb{C}^{r}$. If $\mathcal{V} \circ \mathcal{J}$ is convergent and $\mathcal{J}$ is generically submersive, then $\mathcal{V}$ itself is convergent.

Proof of Theorem 2.1. Restricting the identity (5.2) to the set

$$
\left\{\left(v_{3}\left(z^{\prime}, \xi, \eta\right), \bar{v}_{2}(\xi, \eta)\right):\left(z^{\prime}, \xi, \eta\right) \in\left(\mathbb{C}^{3 n-3}, 0\right)\right\} \subseteq \mathcal{M},
$$

where $v_{j}, j=2,3$, are the Segre sets mappings defined by (3.3), we obtain

$$
\mathcal{R}\left(v_{3}\left(z^{\prime}, \xi, \eta\right),\left(\bar{f}^{\prime} \circ \bar{v}_{2}\right)(\xi, \eta)\right)=\left(\bar{f}_{n} \circ \bar{v}_{2}\right)(\xi, \eta) .
$$

Here, $\mathcal{R}$ is the formal power series defined by (5.1). We would like to apply Lemma 6.1 to the formal equation (6.2) with $y=z^{\prime}, x=\eta, u=\xi, \mathcal{T}(x, u)=$ $\left(\bar{f} \circ \bar{v}_{2}\right)(\xi, \eta), W=(\lambda, \mu), \lambda \in \mathbb{C}^{n-1}, \mu \in \mathbb{C}$ and

$$
\varphi\left((\lambda, \mu) ; \eta, \xi, z^{\prime}\right):=\mathcal{R}\left(v_{3}\left(z^{\prime}, \xi, \eta\right), \lambda\right)-\mu .
$$


For this, one has to check that any derivative of the formal holomorphic power series $\mathbb{C}^{4 n-4} \ni\left(z^{\prime}, \xi, \eta, \lambda\right) \mapsto \mathcal{R}\left(v_{3}\left(z^{\prime}, \xi, \eta\right), \lambda\right)$ with respect to $z^{\prime}$ evaluated at $z^{\prime}=\eta$ is convergent with respect to the variables $\lambda, \xi$ and $\eta$. All these derivatives involve derivatives of $v_{3}$ at $z^{\prime}=\eta$ (which are convergent) and derivatives of the form $\left[\mathcal{R}_{z^{\gamma}}\left(v_{3}\left(z^{\prime}, \xi, \eta\right), \lambda\right)\right]_{z^{\prime}=\eta}$, for $\gamma \in \mathbb{N}^{n}$. Because of the reality condition (3.2) and the definition of $v_{1}$ and $v_{3}$ given by (3.3), we have

$$
v_{3}(\eta, \xi, \eta)=v_{1}(\eta)
$$

This implies that for each $\gamma \in \mathbb{N}^{n}$, we have

$$
\left[\mathcal{R}_{z^{\gamma}}\left(v_{3}\left(z^{\prime}, \xi, \eta\right), \lambda\right)\right]_{z^{\prime}=\eta}=\mathcal{R}_{z^{\gamma}}\left(v_{1}(\eta), \lambda\right),
$$

with the right-hand side being convergent in $(\eta, \lambda)$ by Proposition 5.1. Thus, by Lemma 6.1 , we have, for any positive integer $e$, a convergent power series mapping, denoted $\mathcal{T}^{e}(\xi, \eta)=\left(\mathcal{T}^{\prime} e(\xi, \eta), \mathcal{T}_{n}^{e}(\xi, \eta)\right) \in \mathbb{C}^{n-1} \times \mathbb{C}$, which agree up to order $e$ with $\left(\bar{f} \circ \bar{v}_{2}\right)(\xi, \eta)$ and such that

$$
\mathcal{R}\left(v_{3}\left(z^{\prime}, \xi, \eta\right), \mathcal{T}^{\prime} e(\xi, \eta)\right)=\mathcal{T}_{n}^{e}(\xi, \eta) \text {, in } \mathbb{C}[[z, \xi, \eta]] .
$$

Since $\mathcal{T}^{e}(\xi, \eta)$ is a convergent power series mapping, in order to show that $\mathcal{R}(z, \lambda) \in \mathbb{C}\{z, \lambda\}$, it suffices to show by Proposition 6.2 that for $e$ large enough the generic rank of the holomorphic map

$$
\left(\mathbb{C}^{3 n-3}, 0\right) \ni\left(z^{\prime}, \xi, \eta\right) \mapsto\left(v_{3}\left(z^{\prime}, \xi, \eta\right), \mathcal{T}^{\prime} e(\xi, \eta)\right) \in \mathbb{C}^{2 n-1}
$$

is $2 n-1$. For this, note that since $M$ is minimal at 0 , the map $\bar{v}_{2}$ is of generic rank $n$, and thus, by the form of $v_{3}$ given in $(3.3)$, the holomorphic map $\left(\mathbb{C}^{3 n-3}, 0\right) \ni$ $\left(z^{\prime}, \xi, \eta\right) \mapsto\left(v_{3}\left(z^{\prime}, \xi, \eta\right), \bar{v}_{2}(\xi, \eta)\right) \in \mathcal{M}$ is of generic rank $2 n-1$ (see [2]). Moreover, since $f$ is invertible, we have $\operatorname{det}\left(\frac{\partial f^{\prime}}{\partial z^{\prime}}(0)\right) \neq 0$, which implies that the rank of the formal map $\mathcal{M} \ni(z, w) \mapsto\left(z, \bar{f}^{\prime}(w)\right)$ is $2 n-1$ (at the origin). From this, we see that the formal map $\left(\mathbb{C}^{3 n-3}, 0\right) \ni\left(z^{\prime}, \xi, \eta\right) \mapsto\left(v_{3}\left(z^{\prime}, \xi, \eta\right),\left(\bar{f}^{\prime} \circ \bar{v}_{2}\right)(\xi, \eta)\right)$ has rank $2 n-1$. (The rank of such a formal map is its rank in the quotient field of $\mathbb{C}\left[\left[z^{\prime}, \xi, \eta\right]\right]$.) Since $\mathcal{T}^{e}(\xi, \eta)$ agrees up to order $e$ with $\left(\bar{f} \circ \bar{v}_{2}\right)(\xi, \eta)$, we obtain that for $e$ large enough, the mapping (6.4) is of generic rank $2 n-1$. This completes the proof of Theorem 2.1.

Remark 3. When the target hypersurface $M^{\prime}$ is given in normal coordinates i.e., $\bar{\Phi}^{\prime}(\omega, 0) \equiv \omega_{n}$, then the normal component $f_{n}$ of a formal biholomorphism $f:(M, 0) \rightarrow\left(M^{\prime}, 0\right)$ is convergent provided that the source hypersurface $M$ is minimal. Indeed, this follows by taking $\lambda=0$ in Theorem 2.1.

Proof of Theorem 2.2. By the Taylor expansion (5.3) and by Theorem 2.1, we obtain that all the $\phi_{\alpha}^{\prime} \circ f$ are convergent in a common neighborhood $U$ of $0 \in \mathbb{C}^{n}$. Since $M^{\prime}$ is holomorphically nondegenerate, by $[7,16]$, there exists $\phi_{\beta^{1}}^{\prime}(\omega), \ldots, \phi_{\beta^{n}}^{\prime}(\omega), \beta^{i} \in \mathbb{N}^{n-1}, i=1, \ldots, n$, such that

$$
\operatorname{det}\left[\frac{\partial \phi_{\beta^{i}}^{\prime}}{\partial \omega_{j}}(\omega)\right]_{1 \leq i, j \leq n} \not \equiv 0 \text {. }
$$


Since $f$ is a formal biholomorphism, this implies that

$$
\operatorname{det}\left[\frac{\partial \phi_{\beta^{i}}^{\prime}}{\partial \omega_{j}}(f(z))\right]_{1 \leq i, j \leq n} \not \equiv 0,
$$

as a formal power series in $z$. Put $\psi_{i}(z):=\left(\phi_{\beta^{i}}^{\prime} \circ f\right)(z)$ and $R_{i}(z, \omega):=\phi_{\beta^{i}}^{\prime}(\omega)-$ $\psi_{i}(z), i=1, \ldots, n$. Observe that since $\psi_{i}(z)$ is convergent, $R_{i}(z, \omega) \in \mathbb{C}\{z, \omega\}$ for $i=1, \ldots, n$. Moreover, since $R_{i}(z, f(z))=0, i=1, \ldots, n$, in $\mathbb{C}[[z]]$, by (6.5), we may apply Proposition 4.2 to conclude that $f$ is convergent.

\section{Transcendence degree and partial convergence of formal maps}

In this last section, we want to indicate how Theorem 2.1 can be viewed as a result of partial convergence for formal biholomorphic mappings of real analytic hypersurfaces. Before explaining what we mean by this, we need to recall the following. If $M$ is a real analytic hypersurface in $\mathbb{C}^{n}$ and $p \in M$, let $\mathbb{K}(p)$ be the quotient field of $\mathbb{C}\{z-p\}$, and $H(M, p)$ be the vector space over $\mathbb{K}(p)$ consisting of the germs at $p$ of $(1,0)$ vector fields, with meromorphic coefficients, tangent to $M$ (near $p$ ). We then define the degeneracy of $M$ at $p$, denoted $d(M, p)$, to be the dimension of $H(M, p)$ over $\mathbb{K}(p)$. It is shown in [7] that the mapping $M \ni p \mapsto d(M, p) \in\{0, \ldots, n\}$ is constant on any connected component of $M$. Thus, if $M$ is a connected real analytic hypersurface, one can define its degeneracy $d(M)$ to be the degeneracy $d(M, q)$ at any point $q \in M$. Observe that the germ $(M, p), p \in M$, is holomorphically nondegenerate if and only if $d(M)=d(M, p)=0$.

Theorem 2.1 gives the following result of partial convergence for formal biholomorphic mappings of real analytic hypersurfaces. By this, we mean that we have the following.

Theorem 7.1. Let $f:(M, 0) \rightarrow\left(M^{\prime}, 0\right)$ be a formal biholomorphism between two germs at 0 of smooth real real-analytic hypersurfaces in $\mathbb{C}^{n}$. Assume that $M$ is minimal at 0 and let $d\left(M^{\prime}\right)$ be the degeneracy of the germ $\left(M^{\prime}, 0\right)$. Then, there exists $g(\omega)=\left(g_{1}(\omega), \ldots, g_{n-d\left(M^{\prime}\right)}(\omega)\right) \in(\mathbb{C}\{\omega\})^{n-d\left(M^{\prime}\right)}, \omega \in \mathbb{C}^{n}$, of generic rank $n-d\left(M^{\prime}\right)$ such that the mapping $g \circ f$ is convergent.

Proof. We again use the notations of $\S 2$. As in the proof of Theorem 2.2, we have, using the expansion (5.3),

$$
\bar{\Phi}^{\prime}(f(z), \lambda)=\sum_{\beta \in \mathbb{N}^{n-1}}\left(\phi_{\beta}^{\prime} \circ f\right)(z) \lambda^{\beta} .
$$

Thus, we know, by Theorem 2.1, that for any multi-index $\beta \in \mathbb{N}^{n-1},\left(\phi_{\beta}^{\prime} \circ f\right)(z)$ is convergent in some neighborhood $U$ of 0 in $\mathbb{C}^{n}$. We choose $\phi_{\beta^{1}}^{\prime}(\omega), \ldots, \phi_{\beta^{r}}^{\prime}(\omega)$, $r=n-d\left(M^{\prime}\right)$, of generic maximal rank equal to $n-d\left(M^{\prime}\right)$ in a neighborhood $U^{\prime}$ of 0 in $\mathbb{C}^{n}$ (see [7]). Then, if we define $g_{j}(\omega)=\phi_{\beta^{j}}^{\prime}(\omega), j=1, \ldots, n-d\left(M^{\prime}\right)$, we obtain the desired statement of the Theorem. 
Remark 4. One should observe that the convergent power series mapping $g$ in Theorem 7.1 is obtained in a constructive way from the target manifold $M^{\prime}$. Indeed, this is a consequence of the statement of Theorem 2.1.

Now, we want to make explicit links with the notion of transcendence degree introduced in [10] in the $\mathcal{C}^{\infty}$ mapping problem. For this, we first set the corresponding definitions in the formal case.

Definition 7.1. Let $\mathcal{H}:\left(\mathbb{C}^{N}, 0\right) \rightarrow\left(\mathbb{C}^{N^{\prime}}, 0\right)$ be a formal (holomorphic) mapping, and $V$ be a complex analytic set through the origin in $\mathbb{C}^{N} \times \mathbb{C}^{N^{\prime}}$. Assume that $V$ is given near the origin in $\mathbb{C}^{N+N^{\prime}}$ by

$$
V=\left\{(x, y) \in \mathbb{C}^{N} \times \mathbb{C}^{N^{\prime}}: b_{1}(x, y)=\ldots=b_{q}(x, y)=0\right\},
$$

$b_{i}(x, y) \in \mathbb{C}\{x, y\}, i=1, \ldots, q$. Then, the graph of $\mathcal{H}$ is said to be formally contained in $V$ if $b_{1}(x, \mathcal{H}(x))=\ldots=b_{q}(x, \mathcal{H}(x))=0$ in $\mathbb{C}[[x]]$.

It follows from the Nullstellensatz that this definition is independent of the choice of the defining functions $\left(b_{i}\right)$ for $V$.

Definition 7.2. Let $\mathcal{H}:\left(\mathbb{C}^{N}, 0\right) \rightarrow\left(\mathbb{C}^{N^{\prime}}, 0\right)$ be a formal holomorphic mapping. Let $V_{\mathcal{H}}$ be the germ of the complex analytic set through the origin in $\mathbb{C}^{N+N^{\prime}}$ defined as the intersection of all the complex analytic sets through the origin in $\mathbb{C}^{N+N^{\prime}}$ which formally contain the graph of $\mathcal{H}$. Then the transcendence degree of $\mathcal{H}$ is the nonnegative integer $\operatorname{dim}_{\mathbb{C}} V_{\mathcal{H}}-N$.

This definition is motivated by the following result.

Proposition 7.2. Let $\mathcal{H}:\left(\mathbb{C}^{N}, 0\right) \rightarrow\left(\mathbb{C}^{N^{\prime}}, 0\right)$ be a formal holomorphic mapping. Then, the following conditions are equivalent:

i) $\mathcal{H}$ is convergent.

ii) The transcendence degree of $\mathcal{H}$ is zero.

Proof. The implication i) $\Rightarrow$ ii) is clear. The other implication is equivalent to the following proposition.

Proposition 7.3. Let $\mathcal{H}:\left(\mathbb{C}^{N}, 0\right) \rightarrow\left(\mathbb{C}^{N^{\prime}}, 0\right)$ be a formal (holomorphic) mapping. If there exists a germ at 0 of a complex analytic set $V \subset \mathbb{C}^{N+N^{\prime}}$ which formally contains the graph of $\mathcal{H}$ with $\operatorname{dim}_{\mathbb{C}} V=N$, then $\mathcal{H}$ is convergent.

Proof. Let $V$ be as in the proposition. We can assume that, near $0 \in \mathbb{C}^{N+N^{\prime}}$,

$$
V=\left\{(x, y) \in \mathbb{C}^{N} \times \mathbb{C}^{N^{\prime}}: b_{1}(x, y)=\ldots=b_{p}(x, y)=0\right\},
$$

where each $b_{j}(x, y) \in \mathbb{C}\{x, y\}$. To this complex analytic set $V$, as is customary, we associate the following ideal of $\mathbb{C}\{x, y\}$ defined by

$$
\mathcal{I}(V)=\{s \in \mathbb{C}\{x, y\}: s \text { vanishes on } V\} .
$$

By the Noetherian property, we can assume that $\mathcal{I}(V)$ is generated by a family $\left(h_{i}(x, y)\right)_{i=1, \ldots, k} \subseteq \mathbb{C}\{x, y\}$. Furthermore, by the Nullstellensatz [12, 17], for $i=$ $1, \ldots, k$, there exists an integer $\mu_{i}$ such that $h_{i}^{\mu_{i}}(x, y)$ is in the ideal generated by 
the $b_{j}(x, y), j=1, \ldots, p$. This implies that for $i=1, \ldots, k, h_{i}(x, \mathcal{H}(x))=0$ in $\mathbb{C}[[x]]$. The following result, a consequence of the Artin approximation theorem, is contained in [17] (p.63). Assume that the height of the ideal $\mathcal{I}(V)$ (generated by the family $\left(h_{j}(x, y)\right)_{1 \leq j \leq k}$ in $\left.\mathbb{C}\{x, y\}\right)$ is equal to $N^{\prime}$. Then, any formal solution $\mathcal{Y}(x) \in(\mathbb{C}[[x]])^{N^{\prime}}, \mathcal{Y}(0)=0$, of the system $h_{1}(x, y)=\ldots=h_{k}(x, y)=0$ (in the unknown $y$ ) is convergent. Thus, to obtain the convergence of our original formal power series $\mathcal{H}$, it suffices to check that the height of $\mathcal{I}(V)$ is equal to $N^{\prime}$. Since $\mathbb{C}\{x, y\}$ is a local regular ring of Krull dimension $N+N^{\prime}$, by Proposition 6.12 , p. 22 of [17], we have the formula

$$
\operatorname{height}(\mathcal{I}(V))+\operatorname{dim} \mathbb{C}\{x, y\} / \mathcal{I}(V)=N+N^{\prime},
$$

where $\operatorname{dim} \mathbb{C}\{x, y\} / \mathcal{I}(V)$ is the Krull dimension of the ring $\mathbb{C}\{x, y\} / \mathcal{I}(V)$. Since the Krull dimension of such a ring coincides with the dimension of the complex analytic set $V$ ( $c f$. [12], p. 226, Proposition 1), which is, here, equal to $N$, we obtain that the height of $\mathcal{I}(V)$ is $N^{\prime}$. This completes the proof of Proposition 7.3, and hence, the proof of Proposition 7.2.

With these tools at our disposal, we can now state a result which follows from Theorem 7.1.

Corollary 7.4. Let $f:(M, 0) \rightarrow\left(M^{\prime}, 0\right)$ be a formal biholomorphism between two germs at 0 of smooth real real-analytic hypersurfaces in $\mathbb{C}^{n}$. Assume that $M$ is minimal at 0 and denote by $\mathcal{D}_{f}$ the transcendence degree of the map $f$. Then, $\mathcal{D}_{f} \leq d\left(M^{\prime}\right)$, where $d\left(M^{\prime}\right)$ is the degeneracy of $M^{\prime}$. In other words, there exists a complex analytic set of (pure) dimension $n+d\left(M^{\prime}\right)$ which formally contains the graph of $f$.

Proof. By Theorem 7.1, there exists a convergent power series mapping $g(\omega)=$ $\left(g_{1}(\omega), \ldots, g_{n-d\left(M^{\prime}\right)}(\omega)\right) \in(\mathbb{C}\{\omega\})^{n-d\left(M^{\prime}\right)}$ such that for each $j=1, \ldots, r$, $\delta_{j}(z):=\left(g_{j} \circ f\right)(z)$ is convergent, $r=n-d\left(M^{\prime}\right)$. Then, the graph of $f$ is formally contained in the complex analytic set

$$
A=\left\{(z, \omega) \in\left(\mathbb{C}^{2 n}, 0\right): g_{1}(\omega)-\delta_{1}(z)=\ldots=g_{r}(\omega)-\delta_{r}(z)=0\right\} .
$$

Let $A=\cup_{i=1}^{k} \Gamma_{i}$ be the decomposition of $A$ into irreducible components. For any positive integer $\sigma$, one can find, according to the Artin approximation theorem [1], a convergent power series mapping $f^{\sigma}(z) \in(\mathbb{C}\{z\})^{n}$ defined in some small neighborhood $U^{\sigma}$ of 0 in $\mathbb{C}^{n}$, which agrees with $f(z)$ up to order $\sigma$ (at 0 ) and such that the graph of $f^{\sigma}$, denoted $G\left(f^{\sigma}\right)$, is contained in $A$. Since $G\left(f^{\sigma}\right)$ is contained in $A$, it must be contained in an irreducible component of $A$. Thus, by the pigeonhole principle, at least one subsequence of $\left(f^{\sigma}\right)_{\sigma \in \mathbb{N}^{*}}$ is contained in one of such irreducible components, say $\Gamma_{1}$. There is no loss of generality in assuming that such a subsequence is $\left(f^{\sigma}\right)_{\sigma \in \mathbb{N}^{*}}$ itself. We first observe that this implies that the graph of $f$ is formally contained in $\Gamma_{1}$. Moreover, since $f$ is a formal biholomorphism, the family $\left(f^{\sigma}\right)_{\sigma \in \mathbb{N}^{*}}$ is also a family of local biholomorphisms. In particular, this implies that the generic rank of the family of 
holomorphic functions

$$
\left(\left(g_{i} \circ f^{1}\right)(z)\right)_{1 \leq i \leq r},
$$

is $r$. As a consequence, if $z_{0}$ is close enough to 0 in $\mathbb{C}^{n}$ and is chosen so that the rank of the preceding family at $z_{0}$ equals $r$, the implicit function theorem shows that $A$ is an $n+d\left(M^{\prime}\right)$-dimensional complex submanifold near $\left(z_{0}, f^{1}\left(z_{0}\right)\right) \in$ $\Gamma_{1}$. Since $\Gamma_{1}$ is irreducible, it is pure-dimensional; thus $\Gamma_{1}$ is an $n+d\left(M^{\prime}\right)$ pure-dimensional complex analytic set formally containing the graph of $f$. By definition of the transcendence degree, this implies that $\mathcal{D}_{f} \leq d\left(M^{\prime}\right)$.

The following example illustrates the applications of Theorem 7.1 and Corollary 7.4.

Example 1. Let $M=M^{\prime}$ be the minimal real algebraic hypersurface through the origin in $\mathbb{C}^{3}$ given by

$$
\operatorname{Im} z_{3}=\left|z_{1} z_{2}\right|^{2} .
$$

Here, $M$ is holomorphically degenerate and its degeneracy $d(M)$ is equal to 1 . Consider the following formal biholomorphic self-map of $M$ :

$$
f_{h}: \mathbb{C}^{3} \ni\left(z_{1}, z_{2}, z_{3}\right) \mapsto\left(z_{1} e^{h(z)}, z_{2} e^{-h(z)}, z_{3}\right) \in \mathbb{C}^{3},
$$

where $h(z)=h\left(z_{1}, z_{2}, z_{3}\right)$ is any non-convergent formal power series vanishing at the origin. Observe that Theorem 2.1 gives in this example that for any formal biholomorphic self-map $f=\left(f_{1}, f_{2}, f_{3}\right)$ of $M$, the product $f_{1} f_{2}$ and the third component $f_{3}$ are necessarily convergent. Observe in this example that the first two components of $f_{h}$ are not convergent, but that the transcendence degree of the map $f_{h}$ is actually $1=d(M)$. Indeed, the graph of $f_{h}$ is formally contained in the complex analytic set of dimension 4

$$
V=\left\{(z, \omega) \in \mathbb{C}^{3} \times \mathbb{C}^{3}: \omega_{1} \omega_{2}=z_{1} z_{2}, \omega_{3}=z_{3}\right\},
$$

and cannot be formally contained in a complex analytic set of dimension 3 , since otherwise $f_{h}$ would be convergent by Proposition 7.3.

We conclude by observing that Theorem 2.2 can be regarded as a direct consequence of Corollary 7.4. Indeed, when $M^{\prime}$ is holomorphically nondegenerate, as mentioned above, $d\left(M^{\prime}\right)=0$ and hence $\mathcal{D}_{f}=0$ by Corollary 7.4. It then follows from Proposition 7.2 that $f$ is convergent in that case. We should also mention that Theorem 2.2, Theorem 7.1 and Corollary 7.4 are all consequences of our main result, namely Theorem 2.1 .

\section{Acknowledgments}

I would like to thank Makhlouf Derridj for his interest in this work and Vincent Thilliez for calling my attention to the paper [15]. I wish also to address special thanks to Salah Baouendi and Linda Rothschild for many simplifying remarks and suggestions. 


\section{References}

[1] M. Artin, On the solution of analytic equations, Invent. Math. 5 (1968), 277-291.

[2] M.S. Baouendi, P. Ebenfelt, and L.P. Rothschild, Real submanifolds in complex space and their mappings, Princeton University Press, N.J., 1999.

[3] _ Parametrization of local biholomorphisms of real analytic hypersurfaces, Asian J. Math. 1 (1997), 1-16.

[4] _ Rational dependence of smooth and analytic CR mappings on their jets, Math. Ann. 315 (1999), 205-249.

[5] - Convergence and finite determination of formal CR mappings, J. Amer. Math. Soc., to appear, 1999.

[6] M.S. Baouendi, X. Huang, and L.P. Rothschild, Regularity of CR mappings between algebraic hypersurfaces, Invent. Math. 125 (1996), 13-36.

[7] M.S. Baouendi and L.P. Rothschild, Mappings of real algebraic hypersurfaces, J. Amer. Math. Soc. 8 (1995), 997-1015.

[8] E. Bierstone and P.D. Milman, Semianalytic and subanalytic sets, Inst. Hautes Études Sci. Publ. Math. 67 (1988), 5-42.

[9] S.S. Chern and J.K. Moser, Real hypersurfaces in complex manifolds, Acta Math. 133 (1974) 219-271.

[10] B. Coupet, S. Pinchuk, and A. Sukhov, Analyticité des applications CR, C. R. Acad. Sci. Paris Sér. I Math. 329 (1999), 489-494.

[11] X. Huang, Schwarz reflection principle in complex spaces of dimension two. Comm. Partial Differential Equations 21 (1996), 1781-1829.

[12] S. Lojasiewicz, Introduction to complex analytic geometry, Birkhäuser Verlag, Basel, 1991.

[13] N. Mir, Germs of holomorphic mappings between real algebraic hypersurfaces. Ann. Inst. Fourier (Grenoble) 48 (1998), 1025-1043.

[14] - On the convergence of formal mappings, preprint, 1999.

[15] T.S. Neelon, On solutions of real analytic equations, Proc. Amer. Math. Soc. 125 (1997), 2531-2535.

[16] N. Stanton, Infinitesimal CR automorphisms of real hypersurfaces, Amer. J. Math 118 (1996), 209-233.

[17] J.C. Tougeron, Idéaux de fonctions différentiables, Springer-Verlag, Berlin-New York, 1972.

[18] S. Webster, On the mapping problem for algebraic real hypersurfaces. Invent. Math. 43 (1977), 53-68.

[19] O. Zariski and P. Samuel, Commutative algebra, Vols. 1, 2, D. Van Nostrand Co., Princeton, New Jersey, 1958.

Université de Rouen, Laboratoire de Mathématiques Raphä̈l Salem, CNRS, Site Colbert 76821 Mont Saint Aignan France

E-mail address: Nordine.Mir@univ-rouen.fr 